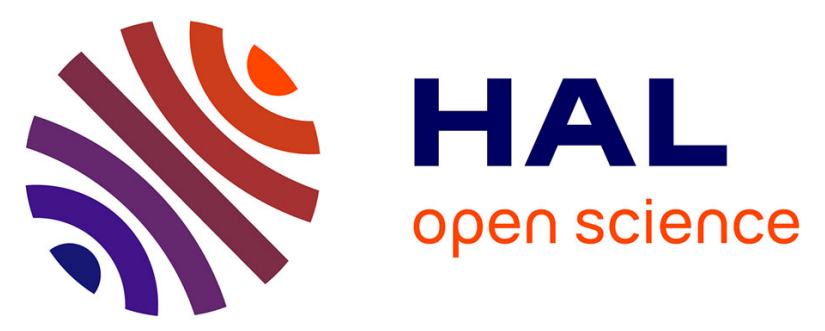

\title{
Quantitative characterization of tissue microstructure in concentrated cell pellet biophantoms based on the structure factor model
}

\author{
Emilie Franceschini, Romain de Monchy, Jonathan Mamou
}

\section{To cite this version:}

Emilie Franceschini, Romain de Monchy, Jonathan Mamou. Quantitative characterization of tissue microstructure in concentrated cell pellet biophantoms based on the structure factor model. IEEE Transactions on Ultrasonics, Ferroelectrics and Frequency Control, 2016, 10.1109/TUFFC.2016.2549273 . hal-01297168

\section{HAL Id: hal-01297168 \\ https://hal.science/hal-01297168}

Submitted on 3 Apr 2016

HAL is a multi-disciplinary open access archive for the deposit and dissemination of scientific research documents, whether they are published or not. The documents may come from teaching and research institutions in France or abroad, or from public or private research centers.
L'archive ouverte pluridisciplinaire HAL, est destinée au dépôt et à la diffusion de documents scientifiques de niveau recherche, publiés ou non, émanant des établissements d'enseignement et de recherche français ou étrangers, des laboratoires publics ou privés. 


\title{
Quantitative characterization of tissue
}

\section{microstructure in concentrated cell pellet}

\section{biophantoms based on the structure factor}

\author{
model
}

Emilie Franceschini, Member IEEE, Romain de Monchy, and Jonathan Mamou, Senior Member IEEE

\begin{abstract}
Quantitative ultrasound (QUS) methods based on the backscatter coefficient (BSC) are typically model-based. The BSC is estimated from experiments and is fit to a model. The fit parameters often are termed QUS estimates and are used to characterize the scattering properties of the tissue under investigation. Nevertheless, for physical interpretation of QUS estimates to be accurate, the scattering model chosen must also be accurate. The goal of this work was to investigate the use of the structure factor model (SFM) to take into account coherent scattering from dense concentration of scatterers. The study focuses on comparing the performance of two sparse models (fluid-filled sphere and Gaussian) and one concentrated model (SFM) to estimate QUS parameters from simulations and cell pellet biophantoms with a range of scatterer volume fractions. Results demonstrated the superiority of the SFM for all investigated volume fractions (i.e., from 0.006 to 0.30). In particular, the sparse models underestimated scatterer size and overestimated acoustic concentration when the volume fraction was greater than 0.12 . In addition, the SFM has the ability to provide the volume fraction and the relative impedance contrast (instead of only the acoustic concentration provided by the sparse models), which could have a great benefit for tissue characterization. This study demonstrates that the SFM could prove to be an invaluable tool for QUS and could help to more accurately characterize tissue from ultrasound measurements.
\end{abstract}

This work was supported by the French Agence Nationale de la Recherche (ANR) under grant ANR Tecsan 11-008-01 and under the A*MIDEX project (ANR-11-IDEX-0001-02) funded by the Investissements d'Avenir French Government program. This work was also supported in part by Riverside Research, Internal Research and Development.

E. Franceschini and Romain de Monchy are with the Laboratoire de Mécanique et d'Acoustique LMA - CNRS UPR 7051, Aix-Marseille University, Centrale Marseille, F-13402 Marseille Cedex 20, France (e-mail: franceschini@1ma.cnrs-mrs.fr).

J. Mamou is with the F. L. Lizzi Center for Biomedical Engineering, Riverside Research, New York, NY, USA. 


\section{Quantitative characterization of tissue}

\section{microstructure in concentrated cell pellet}

\section{biophantoms based on the structure factor}

\section{model}

\section{INTRODUCTION}

Quantitative ultrasound (QUS) techniques based on the parametrization of the backscatter coefficient (BSC) are now routinely used to characterize tissue. Specifically, a scattering model is fit to the estimated BSC and the fit parameters can provide a meaningful description of the tissue microstructure (e.g., scatterer size, shape, scattering strength, spatial organization, etc.) provided that the chosen scattering model is accurate for the tissue under investigation. One of the most popular scattering models is the spherical Gaussian model (SGM) developed by Lizzi et al. [1], [2]. This model describes tissue as a random medium composed of spherical structures having continuous spherical impedance fluctuation following a spherical Gaussian curve. One of the key features of the SGM is that fitting is computationally efficient [3] and yields two QUS estimates describing tissue microstructure: the average effective scatterer size and the so-called acoustic concentration (i.e., the product of the scatterer number density and the square of the relative impedance difference between the scatterers and the surrounding medium). A second class of theoretical scattering models describes tissue as an ensemble of discrete scatterers. The discretescattering approaches assume that the cells are the dominant source of scattering: a fluid-filled sphere models the entire cell or the nucleus [12], [13] or two concentric spheres aim to model the cytoplasm and the nucleus [12]-[15]. Both classes of scattering models and their QUS estimates have been sucessfully used in a wide range of theoretical, preclinical, and clinical studies. For example, QUS studies have been performed to assess tumors in the eye [4], prostate [5], breast [3], [6], lymph nodes [7], [8], fatty liver [9] [10] and thyroid [11].

In all these aforementioned models, i.e., the SGM, the fluid-filled sphere model (FFSM) and the concentric sphere model, the scatterers are assumed to be independently and randomly distributed, such that each scatterer individually contributes to the backscattered power. As a result, there is no deterministic phase relationship between the waves scattered by individual scatterers, a condition known as "incoherent scattering". Therefore, the BSC is proportional to the scatterer number density. This relationship has been used to compute estimates of scatterer size and acoustic concentration.

However, despite the notable successes of QUS in differentiating between healthy and pathological tissues or in detecting cancerous tumors, establishing a link between the actual cellular structures and the QUS parameters describing the tissue microstructure often is difficult [12], [14]. This difficulty may originate from an unsuitable 
modeling either for the individual cell or for an ensemble of cells, or from a combination of both factors. This work focuses on conditions under which the assumption of incoherent scattering is not fulfilled because of the presence of densely packed scattering sources that introduce correlation between the phases of individually scattered waves. Indeed, the assumption of randomly and independently distributed scatterers may not hold in tissues with densely packed cells [16]. A model adapted to a concentrated medium is the structure factor model (SFM), which has been used for ultrasonic characterization of blood [17], [18]. The SFM is based on the assumption that at high volume fractions, interference effects are mainly caused by correlations among cell positions (i.e., caused by coherent scattering), which are modeled using a structure function [17], [19]. Franceschini and Guillermin [20] conducted ultrasound experiments in the 6-22 MHz frequency range on tissue-mimicking phantoms (i.e., microsphere suspensions) with scatterer volume fractions ranging from 0.01 to 0.25 . (In this paper [20], the volume fraction is defined as a unitless quantity equal to the ratio of the total volume occupied by the scatterers to the total volume of the medium under investigation.) This study revealed that the SFM was more suitable than scattering models that do not account for the structure factor (i.e., the SGM or the discrete scattering model based on a Faran elasticsphere modeling [21]) to explain the BSC magnitude for concentrated media. Han and O'Brien [22] performed measurements in the 11-105 MHz frequency range on cell pellet biophantoms (that consist of living cells embedded in a plasma-thrombin matrix) with two volume fractions: a high volume fraction of approximately 0.74 and a volume fraction sufficiently low that the structure factor is approximately equal to one. Experimental structure factors were deduced from the measured BSCs of two cell volume fractions (one high and one low). The theoretical polydisperse structure factor agreed with the experimental structure factors, demonstrating the major role of scatterer position correlation on concentrated media. However, the approach based on the experimental structure factors proposed in [22] cannot be implemented in clinical applications for tissue characterization because the volume fraction is assumed to be known a priori and the reference measurement on a diluted medium composed of the same cell line is required.

In a recent study [23], the SFM was used to further our understanding of the measured BSCs from cell pellet biophantoms and in the identification of the cell structures responsible for scattering. A parameter-estimation procedure was performed to estimate the scatterer size and relative impedance contrast that could explain the measured BSC from all the studied cell volume fractions using the SFM or the classical FFSM. The scatterer sizes estimated using this parameter estimation procedure were compared to the true cellular features in order to identify the actual scattering. The study revealed that the FFSM is unable to accurately model the complex effects of the change in volume fraction on the BSC, while the polydisperse SFM is fully capable to model these effects. The fluid-sphere radii estimated with the polydisperse SFM match the actual whole-cell radii for the two cell lines tested, showing that the whole cell plays a major role in the BSC behavior in this study.

In this paper, the aim was to evaluate the performance of two sparse models (SGM and FFSM) and one concentrated model (SFM) to estimate QUS parameters using a direct method, which consists in fitting the measured BSC from a tissue to a theoretical BSC. The ability of the SFM to estimate three parameters (i.e., the scatterer size, 
volume fraction and relative impedance contrast) instead of the two usual QUS parameters (i.e., the scatterer size and acoustic concentration) was also investigated. This evaluation of scattering models was conducted on human leukemia K562 cell pellet biophantoms and on computer simulations with a range of scatterer volume fractions. The K562 cell pellet biophantoms are an excellent experimental tool to test the value of scattering models because the biophantom structure is straightforward (i.e., consisting uniquely of cells) and the whole cells were identified to be the sources of scattering [23]. The simulations mimic the experimental work to test our methods and inversion algorithms under controlled conditions. Two distinct methods for estimating scatterer size and volume fraction with the SFM are compared. The first method is more conventional and directly fit the SFM to the experimental BSC, while the second method is original and fits a theoretical structure factor to the experimental structure factor. These two methods are discussed and evaluated on experimental and simulated data.

\section{ULTRASOUND SCATTERING THEORY}

\section{A. Scattering models for sparse media}

SGM and FFSM assume a sparse distribution of scatterers. By considering an ensemble of scatterers differing only in size that are randomly and independently distributed, the theoretical BSC using the SGM or the FFSM can be expressed as the product of the BSC in the Rayleigh limit and the backscatter form factor $F F$ [24] as follows:

$$
\begin{aligned}
B S C_{\text {polyd }}(k) & =n \int_{0}^{\infty} \sigma_{b}(k, x) f(x) \mathrm{d} x \\
& =n \int_{0}^{\infty} \frac{k^{4} V_{s}(x)^{2} \gamma_{z}^{2}}{4 \pi^{2}} F F(k, x) f(x) \mathrm{d} x
\end{aligned}
$$

where $n$ is the scatterer number density, $k$ is the wavenumber, $\sigma_{b}$ is the differential backscattering cross section, $f$ is the probability density function of the scatterer radii denoted $x, V_{s}(x)=4 / 3 \pi x^{3}$ is the scatterer volume and $\gamma_{z}$ is the relative acoustic impedance difference between scatterers and the surrounding tissue $\gamma_{z}=\frac{z_{0}-z}{z}$. The fluid sphere form factor $F F_{\mathrm{FS}}$ assumes that the scatterer is a homogeneous sphere filled with a fluid, whereas the spherical Gaussian form factor $F F_{\mathrm{SG}}$ hypothesizes a continuous Gaussian distribution with spherical symmetry of relative impedance between the scatterer and the surrounding medium. So, in the case of the SGM, the radius $x$ corresponds to an effective scatterer radius. The form factors are defined as:

$$
\begin{aligned}
& F F_{\mathrm{FS}}(k, x)=\left[\frac{3}{2 k x} j_{1}(2 k x)\right]^{2} \quad \text { (fluid sphere), } \\
& F F_{\mathrm{SG}}(k, x)=e^{-0.827 k^{2} x^{2}} \quad \text { (Gaussian), }
\end{aligned}
$$

where $j_{1}$ is the spherical Bessel function of the first kind of order 1. In the case of a monodisperse size distribution, i.e., an ensemble of identical fluid spheres (or an ensemble of identical effective scatterers) of radius $a$, the theoretical BSC using the FFSM (or the SGM, respectively) reduces to:

$$
B S C_{\text {monod }}(k)=n \sigma_{b}(k, a)=n \frac{k^{4} V_{s}(a)^{2} \gamma_{z}^{2}}{4 \pi^{2}} F F(k, a) .
$$




\section{B. The structure factor model (SFM)}

For a dense scattering medium, the increase in correlation among cells induces interference of the scattered waves that can be modeled with the structure function. When considering a polydisperse size distribution, i.e., an ensemble of scatterers differing only in size with radius $x$ and scattering amplitudes $\Phi(k, x)$, the BSC is given by [22, Eq. (12)] [26]:

$$
\begin{aligned}
& B S C_{\text {FFM }_{\text {polyd }}}(k)=n \int_{0}^{\infty}|\Phi(k, x)|^{2} f(x) \mathrm{d} x+ \\
& n \int_{0}^{\infty} \int_{0}^{\infty} \Phi\left(k, x_{1}\right) \Phi\left(k, x_{2}\right) H_{12}(k) f\left(x_{1}\right) f\left(x_{2}\right) \mathrm{d} x_{1} \mathrm{~d} x_{2}
\end{aligned}
$$

where $H_{12}$ is the partial structure function. In the present study, we use the analytical expression of Eq. (4) that exists when the scattering amplitude is derived from the fluid-filled sphere form factor [22, Eq. (A5)]:

$$
\Phi(k, x)=\frac{\gamma_{z}}{4 k}[\sin (2 k x)-2 k x \cos (2 k x)],
$$

and when the scatterer size distribution follows a gamma probability density function [26]:

$$
f_{(\zeta, a)}(x)=\frac{1}{\zeta !}\left(\frac{\zeta+1}{a}\right)^{\zeta+1} x^{\zeta} e^{-(\zeta+1) x / a},
$$

where $a$ is the mean radius and $\zeta$ is the gamma width factor, $\zeta>-1$, which measures the width of the distribution (a large value of $\zeta$ corresponds to a narrow size distribution). In the remainder of the paper, $f$ will always be a gamma probability density function and the subscript $(\zeta, a)$ will be omitted.

The first integral in Eq. (4) represents the incoherent BSC [and is identical to Eq. (1)], whereas the second integral represents the coherent BSC part originating from the spatial correlation in scatterer positions. The structure factor is defined as the ratio between the (total) BSC and the incoherent BSC and can be written as [22, Eq. (13)]:

$$
\begin{aligned}
& S(k)= \\
& 1+\frac{\int_{0}^{\infty} \int_{0}^{\infty} \Phi\left(k, x_{1}\right) \Phi\left(k, x_{2}\right) H_{12}(k) f\left(x_{1}\right) f\left(x_{2}\right) \mathrm{d} x_{1} \mathrm{~d} x_{2}}{\int_{0}^{\infty}|\Phi(k, x)|^{2} f(x) \mathrm{d} x} .
\end{aligned}
$$

In the case of a monodisperse size distribution, i.e., an ensemble of identical fluid spheres of radius $a$, the BSC is reduced to the following expression:

$$
B S C_{\mathrm{SFM}_{\text {monod }}}(k)=n \sigma_{b}(k, a) S(k, a, \phi),
$$

where the analytical expression of the structure factor $S$ can be obtained as described in [20, Eq. (A1)-(A4)] based on [25].

Illustrative theoretical polydisperse structure factors given by Eq. (7) are plotted in Fig. 1 with the K562 structural parameters ( $a=6.44 \mu \mathrm{m}$ and $\zeta=45$; see Section III.A.) for three different volume fractions (i.e., $0.03,0.30$ and 0.74). Also represented in Fig. 1 are the theoretical monodisperse structure factors given in [20, Eq. (A1)-(A4)]. Both monodisperse and polydisperse curves have similar behaviors for scatterer volume fractions less than 0.30 and differ for the largest volume fraction of 0.74 . 


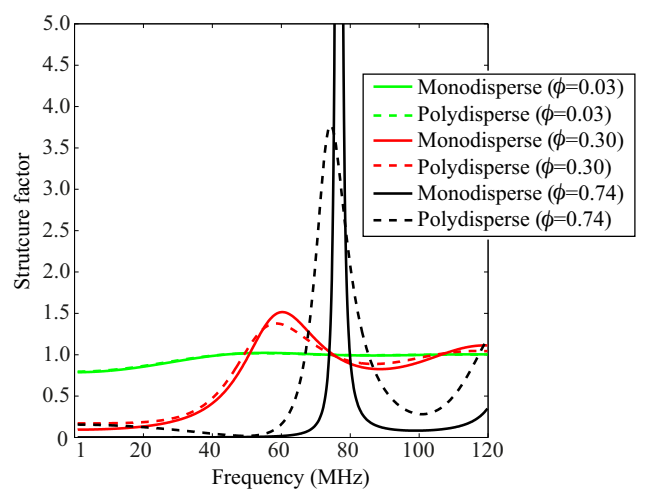

Fig. 1. Comparison between the theoretical monodisperse and polydisperse structure factors for the mean radius $a=6.44 \mu \mathrm{m}$ and the gamma width factor $\zeta=45$ for volume fractions of $0.03,0.30$ and 0.74 .

\section{MATERiAls AND MethodS}

\section{A. Experimental data on cell pellet biophantoms}

Ultrasound backscattered signals were obtained from the experiments described in [23, section III]. These experiments are briefly summarized in this section. Ultrasound experiments were conducted on cell pellet biophantoms that consist of identical human leukemia K562 cells embedded in a plasma-thrombin supportive background with different cell volume fractions of $0.006,0.03,0.06,0.12,0.18,0.24$ and 0.30 . The concentrated biophantoms mimic densely packed cells with controlled cell volume fractions and are simplified versions of real tissue since only a single cell line is considered. Concerning the K562 cell size, measurements were made using a calibrated optical microscope on 200 extracted nuclei and 120 whole cells randomly selected (see [23, Fig. 2]). The radius probability distribution functions for nuclei and whole cells were well approximated by the gamma distribution $\left(r^{2}=0.94\right)$. The mean radius and gamma width factor for whole cells were found to be equal to $a=6.44 \mu \mathrm{m}$ and $\zeta=45$.

Ultrasonic data were acquired using a high frequency ultrasound system (Vevo 770, Visualsonics Inc, Toronto, Canada). Two probes, RMV 710 and RMV 703, were used in B-mode. For the RMV 710 and the RMV 703 probes, the oscillating single-element focused circular transducers had center frequencies of 20 and $30 \mathrm{MHz}$ with $-6 \mathrm{~dB}$ bandwidths of 10-32 and 18-42 MHz, focuses of 15 and $10 \mathrm{~mm}$ and f-numbers of 2.1 and 2.5, respectively. Raw $\mathrm{RF}$ data were digitized at a sampling frequency of $500 \mathrm{MHz}$ (8-bit precision) using a high-speed acquisition card (CS11G8, Gage, Lockport, IL, USA).

During the experiments, the focus of each transducer was positioned $1 \mathrm{~mm}$ below the PBS/cell pellet biophantom interface. A translation stage (Physik Instrument, model M-403.4PD, Karlsruhe, Germany) controlled the probe motion. Six independent B-mode images were constructed from acquired RF echoes by translating the probe every $600 \mu \mathrm{m}$. Examples of these B-mode images obtained with the $20-\mathrm{MHz}$ center frequency probe are provided in [23, Figure 4]. For BSC estimation, 45 independent RF lines were selected, and the length of the signals gated with a rectangular window corresponds to 10 pulse lengths for the RMV710 probe and to 15 pulse lengths for the 

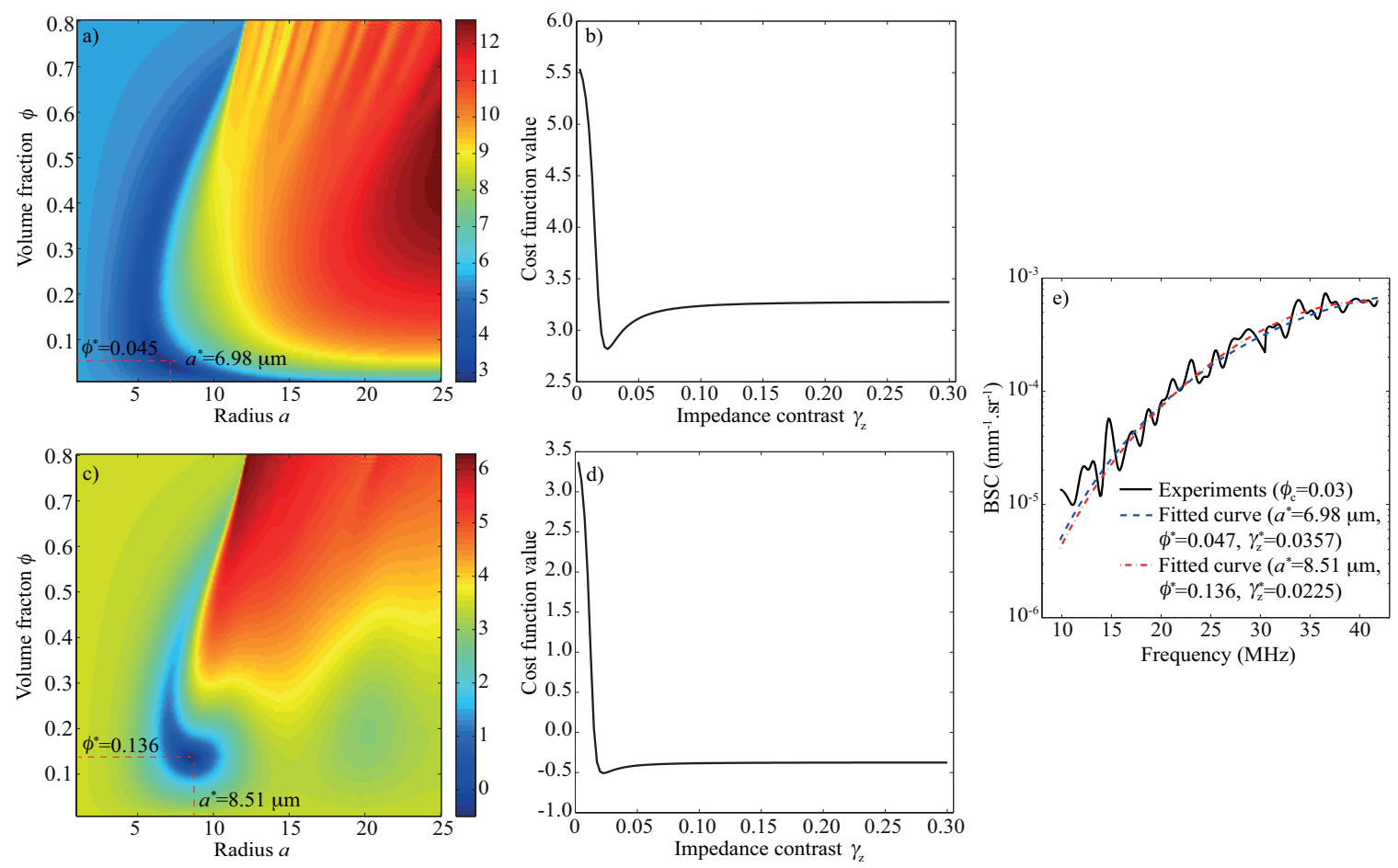

Fig. 2. (a) Logarithm of the cost function $F_{1}(a, \phi)$ for a fixed value of the relative impedance contrast $\gamma_{z}^{*}$ for the monodisperse SFM obtained from the experiment with an actual volume fraction of 0.03. The logarithm is shown here in order to increase the visual contrast. (b) Cost function $F_{1}\left(a^{*}, \phi^{*}, \gamma_{z}\right)$ with the monodisperse SFM for varying values of the relative impedance contrast $\gamma_{z}\left(a^{*}\right.$ and $\phi^{*}$ being calculated for each fixed value of $\gamma_{z}$ ). (c) and (d) Same as (a) and (b) for the estimator 2 with the cost function $F_{2}$. (e) Measured $B S C_{\text {meas }}$ with an actual volume fraction of 0.03 (in solid line) and corresponding fitted curves obtained with the monodisperse SFM using the estimators 1 and 2.

RMV703 probe. The power spectra of the gated RF signals were then averaged to provide $P_{\text {meas }}$. This procedure was repeated for each probe and each biophantom. For each transducer, an experimental $B S C_{\text {meas }}$ was computed from $P_{\text {meas }}$ using the reference phantom method previously described [23, section III-C]. This procedure yielded a $B S C_{\text {meas }}$ for each probe in the same region-of-interest. The two $B S C_{\text {meas }}$ were then combined to yield a single $B S C_{\text {meas }}$ over the combined bandwidth of the two transducers (i.e., $10-42 \mathrm{MHz}$ ).

\section{B. Simulations}

Three-dimensional (3D) simulations were performed to mimic the experimental data obtained from the K562 cell pellet biophantoms. Fluid-filled spheres with a gamma distribution were chosen to model K562 whole cells, with $a$ and $\zeta$ chosen to be equal to $a=6.44 \mu \mathrm{m}$ and $\zeta=45$ to be consistent with the gamma distribution parameters measured optically as shown in [23, Fig. 2(d)]. The size of the simulated volume, $V_{\text {sim }}$, was fixed to $400 \times 400$ $\times 400 \mu \mathrm{m}^{3}$. The spheres were uniformly randomly distributed within $V_{\text {sim }}$ using a Monte Carlo algorithm [27, section II.B.A]. The periodic boundary conditions were imposed during this process, that means the spheres at the boundaries of $V_{\text {sim }}$ could not overlap with the others at the opposite boundaries in order to remove the edge effects. 
The simulated $B S C_{\text {sim }}$ was obtained using the following equation:

$$
B S C_{\text {sim }}(k)=n E\left[\frac{1}{N}\left|\sum_{j=1}^{N} \Phi\left(k, a_{j}\right) e^{-i 2 \mathbf{k} \boldsymbol{r}_{j}}\right|^{2}\right],
$$

where $E$ is the ensemble average, $\Phi$ is the scattering amplitude given in Eq. (5), $r_{j}$ is the location of the $j$ th sphere and $a_{j}$ is the radius of the $j$ th sphere. (Note that the right-hand side of Eq. (9) is technically a function of $\mathbf{k}$ and not $k$, but that dependence disappears because of the ensemble average and the isotropic nature of the random medium considered. For implementation, we specifically averaged along the three main axis directions.) $N$ is the total number of spheres and is obtained by forcing the prescribed volume fraction $\phi$ to satisfy:

$$
\phi=\frac{\sum_{j=1}^{N} \frac{4 \pi}{3} a_{j}^{3}}{V_{\text {sim }}},
$$

and $n$ is therefore the sphere number density equal to $N / V_{\text {sim }}$. The relative acoustic impedance difference was chosen as $\gamma_{z}=0.051$, as estimated by ultrasound in our previous study [23, Table II line 3]. The simulated $B S C_{\text {sim }}$ was computed by averaging over 100 realizations for averaging purposes.

The simulated polydisperse structure factor was then obtained as the ratio between the simulated $B S C_{\text {sim }}$ given in Eq. (9) and the incoherent BSC as obtained in Eq. (1) as follows:

$$
S_{\text {sim }}(k)=\frac{V_{s i m} B S C_{\text {sim }}(k)}{N \int_{0}^{\infty} \sigma_{b}(k, x) f(x) d x} .
$$

For each tested volume fraction, this procedure was repeated ten times to obtain ten simulated $B S C_{\text {sim }}$ and $S_{\text {sim }}$.

\section{QUS parameter estimation from the BSCs}

For each studied volume fraction, the simulated or experimental BSC was fitted to a theoretical model to yield estimates of the scatterer radius $a^{*}$ and acoustic concentration $n_{z}^{*}=\left(n \gamma_{z}^{2}\right)^{*}$ using the SGM or the FFSM, and to yield estimates of $a^{*}, \phi^{*}$ and $\gamma_{z}^{*}$ using the SFM. For comparison with the SGM and the FFSM, the acoustic concentration for the SFM was calculated as:

$$
n_{z}^{*}=\frac{\phi^{*} \gamma_{z}^{* 2}}{E\left[V_{s}\right]}=\frac{\phi^{*} \gamma_{z}^{* 2}}{\frac{(\zeta+3)(\zeta+2)}{(\zeta+1)^{2}} \frac{4 \pi a^{* 3}}{3}}=\frac{\phi^{*} \gamma_{z}^{* 2}}{1.0661 \frac{4 \pi a^{* 3}}{3}},
$$

where the second equality comes from the expression of the third moment of a gamma distribution. (In the case of the polydisperse modeling, the gamma width factor $\zeta$ is assumed to be known a priori and equal to 45 in order to reduce the number of unknown parameters in the inverse problem.) The above optimization procedure is in contrast to our previous study [23, section III-D], where all volume fractions $\phi$ were used simultaneously to estimate $a^{*}$ and $\gamma_{z}^{*}$ and explain the measured BSCs from all the experimental BSC data.

For optimization, two strategies were investigated:

1) Estimator 1: The first optimization strategy determines the QUS estimates by minimizing the relative mean error between the measured $B S C_{\text {meas }}$ and the theoretical $B S C_{\text {theo }}$ :

$$
F_{1}=\sum_{j}\left\|\frac{B S C_{\text {meas }}\left(k_{j}\right)-B S C_{\text {theo }}\left(k_{j}\right)}{B S C_{\text {meas }}\left(k_{j}\right)}\right\|^{2} .
$$


2) Estimator 2: The second optimization strategy determines the QUS estimates by fitting the measured $B S C_{\text {meas }}$ with the theoretical $B S C_{\text {theo }}$, i.e., by minimizing the cost function:

$$
F_{2}=\sum_{j}\left\|B S C_{\text {meas }}\left(k_{j}\right)-B S C_{\text {theo }}\left(k_{j}\right)\right\|^{2} .
$$

The $B S C_{\text {theo }}$ is given by Eqs. (1) and (2) for the SGM and the FFSM and by Eq. (4) for the SFM in the polydisperse case, and given by Eq. (3) for the SGM and the FFSM and by Eq. (8) for the SFM in the monodisperse case. The cost functions were minimized over the 10-42 $\mathrm{MHz}$ range (corresponding to a product $\mathrm{ka}$ ranging from 0.26 to 1.10 , for $a=6.44 \mu \mathrm{m}$ ). Note that because at low frequencies (i.e., near Rayleigh scattering regime), the BSC is nearly proportional to $k^{4}$, estimator 1 essentially weights all frequencies equally and essentially sums up relative errors, whereas estimator 2 penalizes large absolute differences at high frequencies more severely.

For both estimators, the fitting procedure was performed by using the minimization routine "fminsearch" in MATLAB (The MathWorks, Inc., Natick, MA), i.e., a Nelder-Mead simplex method. Both estimators provide empirically cost functions having a unique global minimum as observed by plotting the cost function surfaces. A representative example is shown in Fig. 2 for the monodisperse SFM.

\section{QUS parameter estimation from the experimental structure factors}

The method proposed in section III-C aims to estimate the tissue microstructure from the BSC measurements. An alternative approach was recently proposed by Han and O'Brien [22] based on the experimental structure factor (rather than the entire BSC). To obtain the experimental structure factor, two measurements of biophantoms with two different volume fractions are required: a lower volume fraction for a reference purpose and another volume fraction under consideration. Note that the lower volume fraction is chosen to be sufficiently low such that the structure factor is assumed to be unity (incoherent scattering). The structure factor was obtained experimentally by

$$
S_{\text {meas }}(k)=\frac{n_{\mathrm{L}} B S C_{\text {meas }}(k)}{n B S C_{\text {meas }, \mathrm{L}}(k)}=\frac{\phi_{\mathrm{L}} B S C_{\text {meas }}(k)}{\phi B S C_{\text {meas }, \mathrm{L}}(k)}
$$

where $n_{\mathrm{L}}, \phi_{\mathrm{L}}$ and $B S C_{\text {meas, } \mathrm{L}}$ are the number density, the volume fraction and the measured BSC for the lower volume fraction studied (equal to 0.006 ), and $n, \phi$ and $B S C_{\text {meas }}$ represent the number density, the volume fraction and the measured BSC for the volume fraction under consideration $\left(\phi_{c} \geq 0.03\right)$. To improve the signal-to-noise ratio (SNR), the $B S C_{\text {meas,L }}$ was averaged over the six measurements (corresponding to the six acquired B-mode images), such that the power spectrum $P_{\text {meas }}$ resulted from the average over 600 power spectra of the backscattered RF echoes. The advantage of studying the experimental structure factor rather than the measured BSC is that the effect of spatial scatterer position correlation on scattering can be investigated separately, since the acoustic impedance contrast between the scatterer and the surrounding medium is not affecting the experimental structure factor. The procedure described below consists of assessing the scatterer size and volume fraction using the weighted experimental structure factor defined as $\mathcal{S}_{\text {meas }}(k)=n S_{\text {meas }}(k)=n_{\mathrm{L}} B S C_{\text {meas }}(k) / B S C_{\text {meas, } \mathrm{L}}(k)$, and then of assessing the relative impedance difference using the experimental BSCs. 

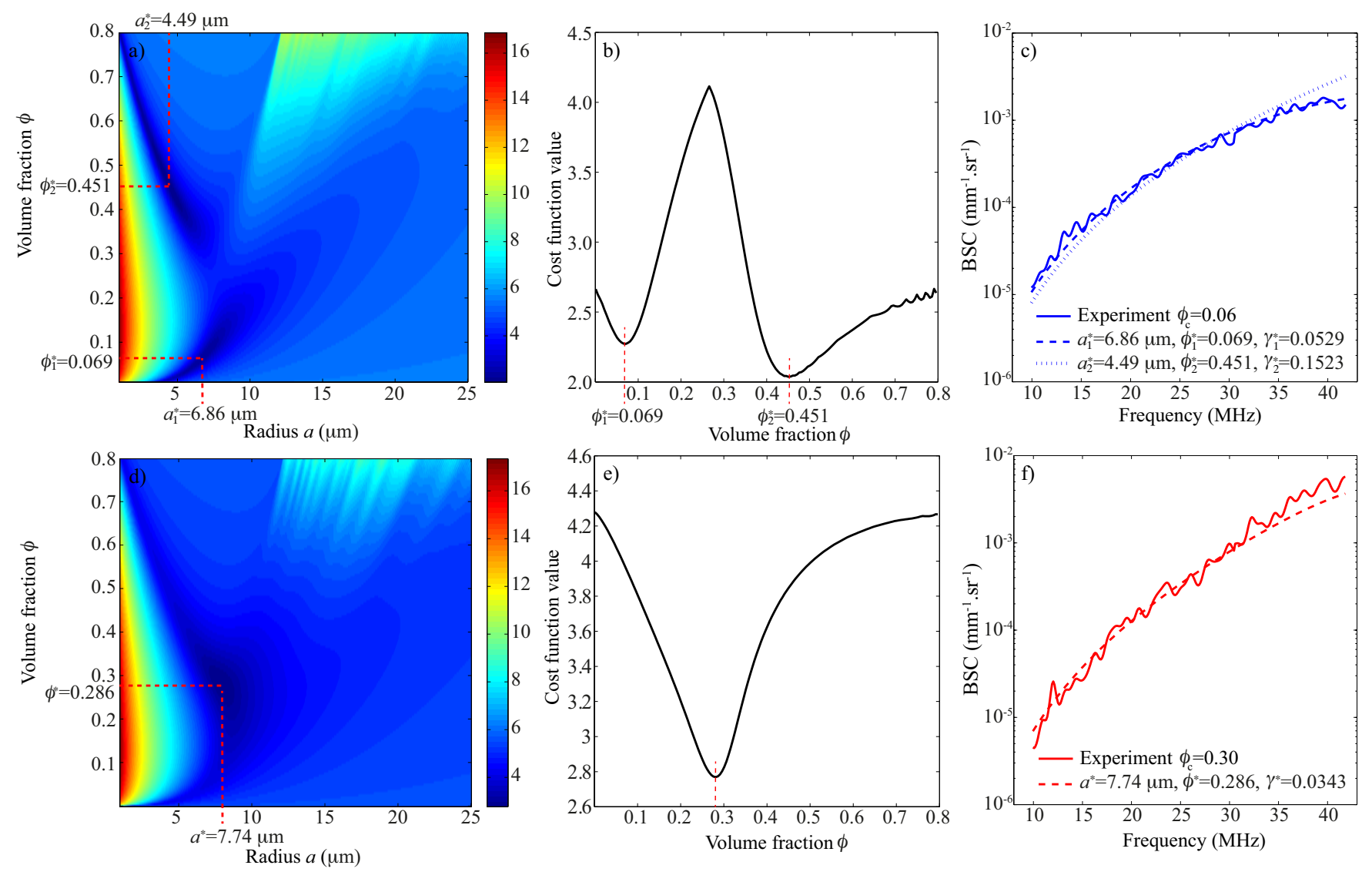

Fig. 3. (a) Logarithm of the cost function $\mathcal{C}(a, \phi)$ showing two minima obtained from the experiments performed with an actual volume fraction of 0.06. (b) Examples of the logarithm of the cost function $\mathcal{C}\left(a^{*}, \phi\right)$ for varying values of $\phi$ ( $a^{*}$ being calculated for each fixed value of $\phi$ ). (c) Measured BSCs with an actual volume fraction of 0.06 (in solid line) and corresponding fitted curves obtained from the combined approach based on both $S_{\text {meas }}$ and $B S C_{\text {meas. }}$ (d) Logarithm of the cost function $\mathcal{C}(a, \phi)$ showing one minimum obtained from the experiments performed with an actual volume fraction of 0.30. (e) Same as (b) for the actual volume fraction of 0.30. (f) Same as (c) for the actual volume fraction of 0.30 .

For each studied volume fraction, the mean radius $a$ and the volume fraction $\phi$ were simultaneously estimated by minimizing the cost function:

$$
\mathcal{C}(a, \phi)=\sum_{j}\left\|\mathcal{S}_{\text {meas }}\left(k_{j}\right)-n S_{\text {theo }}\left(k_{j}\right)\right\|^{2}
$$

over the frequency range from 10 to $42 \mathrm{MHz}$ using "fminsearch". Note that the gamma width factor is assumed to be known a priori $(\zeta=45)$, when using the polydisperse structure factor. The cost function can display two local minima as observed by plotting the cost function surfaces. Figures 3(a) and 3(b) give an example of a cost function surface $\mathcal{C}(a, \phi)$ showing two minima obtained from the experiments performed with an actual volume fraction of 0.06 . Because the cost functions can display two minima, we searched for two estimates $\left(a_{1}^{*}, \phi_{1}^{*}\right)$ and $\left(a_{2}^{*}, \phi_{2}^{*}\right)$ using fminsearch. The structural parameter value obtained $\left(a_{1}^{*}, \phi_{1}^{*}\right)$ [or $\left(a_{2}^{*}, \phi_{2}^{*}\right)$, respectively] corresponds to the minimum 
of the cost function obtained with an initial volume fraction of $\phi^{i n i t}=0.001$ [or $\phi^{i n i t}=0.5$ ] at the beginning of the minimization routine. Note that for true volume fractions greater than 0.18 , the values of $\mathcal{C}\left(a_{1}^{*}, \phi_{1}^{*}\right)$ and $\mathcal{C}\left(a_{2}^{*}, \phi_{2}^{*}\right)$ are equal, i.e., the cost function displays one minimum as shown in the example in Figs. 3(d) and 3(e).

Once the structural parameters $\left(a_{1}^{*}, \phi_{1}^{*}\right)$ and $\left(a_{2}^{*}, \phi_{2}^{*}\right)$ were obtained from the weighted experimental structure factor $\mathcal{S}_{\text {meas }}$, the corresponding relative impedance contrast $\gamma_{z, 1}^{*}$ and $\gamma_{z, 2}^{*}$ were estimated by fitting the measured $B S C_{\text {meas }}$ with the theoretical $B S C_{\mathrm{SFM}}$ given by Eq. (4) in the polydisperse case (or given by Eq. (8) for the SFM in the monodisperse case). Finally, the values of the cost functions $F_{1}\left(a_{1}^{*}, \phi_{1}^{*}, \gamma_{z, 1}^{*}\right)$ and $F_{1}\left(a_{2}^{*}, \phi_{2}^{*}, \gamma_{z, 2}^{*}\right)$ were compared to select the smallest cost function and obtain one set of structural parameters $\left(a^{*}, \phi^{*}, \gamma_{z}^{*}\right)$.

Another simpler procedure consists in estimating the scatterer radius from the experimental structure factor given in Eq. (15), assuming that the volume fraction (as well as the gamma width factor in the polydisperse inversion case) are known a priori. While this approach is unlikely to be used in clinical applications of the SFM for tissue characterization, the results provide interesting insights on the behavior of experimental structure factor in general and are presented in the Appendix.

\section{RESULTS}

\section{A. QUS estimates from the BSCs}

Figure 4 shows the simulated $B S C_{\text {sim }}$ (symbols on the top row) and the experimental $B S C_{\text {meas }}$ (solid lines on the bottom row) in the 10-42 MHz frequency bandwidth. The BSC curves were averaged over the ten simulations for the simulated data and over the six measurements (corresponding to the six acquired B-mode images as described in Section III-A) for the experiments. Also represented are corresponding fitted curves (dashed lines) obtained with the polydisperse and the monodisperse SFM using estimator 1 . The polydisperse and monodisperse SFM gave identical fitting curves that match very well with the measured BSC curves for all the studied volume fractions.

Figures 5 show the three parameters $a^{*}, \phi^{*}$ and $\gamma_{z}^{*}$ estimated simultaneously with the polydisperse and the monodisperse SFM for the simulated data and the experimental data. The QUS parameters were estimated using both estimators 1 and 2 given in Eqs. (13) and (14). These estimated QUS parameters were compared to the true scatterer radius equal to $a=6.44 \mu \mathrm{m}$ as estimated by optical observations, and the true relative impedance difference was equal to $\gamma_{z}=0.051$ as assessed by ultrasound in our previous study [23, Table II line 3]. This relative impedance value was assessed using a specific parameter-estimation procedure to estimate the scatterer size and relative impedance contrast that could explain the measured BSC from all the studied cell volume fractions [23].

For the simulated data (top row of Fig. 5), both estimators gave quasi-identical estimates, except for the smallest true volume fraction of 0.006. When using the polydisperse SFM, the estimated QUS parameters match very well with the true parameters, whatever the studied volume fraction, with relative errors less than $10 \%$ (except for the impedance contrast estimates with the two smallest volume fractions of 0.006 and 0.03 ). When using the monodisperse SFM, the radius and impedance contrast estimates were overestimated and the volume fraction estimates were underestimated. For both polydisperse and monodisperse modelings, it is interesting to note that 
the standard deviations obtained for the impedance contrast estimates are larger for the three smallest true volume fractions.

For the experimental data (bottom row of Fig. 5), the QUS parameter estimates are similar with both estimators (using the polydisperse or monodisperse SFM) for true highest volume fractions $\phi_{c} \geq 0.12$. In contrast, as discussed later in section V-E, the two estimators yielded different QUS estimates for true volume fractions of 0.03 and 0.06. For all investigated volume fractions, radius estimates are overestimated with values comprised between 7.87 and $9.51 \mu \mathrm{m}$ (corresponding to relative errors comprised between $22 \%$ and $47 \%$ ), volume fraction estimates are overestimated, and impedance contrast are underestimated (except for true volume fractions of 0.03 and 0.06 using estimator 1). Nevertheless, excellent correlations $\left(R^{2} \geq 0.88\right)$ were found between the estimated and true cell volume fraction for the polydisperse or monodisperse SFM, whatever the estimator tested.

The two structural parameters $a^{*}$ and $\phi^{*}$ were also estimated with the SFM when the relative impedance contrast is assumed to be known a priori and fixed to $\gamma_{z}=0.051$. Figure 6 summarizes the results. For the simulated data, excellent agreements were obtained between the estimated and true QUS parameters when using the polydisperse modeling, with relative errors less than $3 \%$. The estimated radii (and volume fractions, respectively) were slightly overestimated (and underestimated, respectively) when using the monodisperse modeling, but the relative errors are reasonably small: less than $11 \%$ for the radius and less than $19 \%$ for the volume fraction. For the experimental data, both estimators yield similar QUS parameters for all studied volume fractions, contrary to the results obtained previously in the bottom panel of Fig. 5 where all three QUS parameters were estimated simultaneously. In addition, for true volume fractions smaller than 0.12 , the volume fraction estimates are greatly improved when compared to those obtained previously when the relative impedance contrast was estimated together with the volume fraction and the scatterer radius. For example, when using the monodisperse SFM with the estimator 2, the relative errors for the volume fractions are comprised between 0.9 and $27 \%$ (against relative errors comprised between 52 and $431 \%$ in the bottom panel of Fig. 5).

Thereafter, we compared the radius $a^{*}$ and the acoustic concentration $\left(n_{z}\right)^{*}$ estimated by the three scattering models: the SGM, the FFSM and the SFM (Fig. 7). Both polydisperse and monodisperse modeling gave similar results for the three scattering models (data not shown in the monodisperse case). The three models gave similar scatterer radius and acoustic concentration estimates for the smallest $\phi_{c} \leq 0.06$, but the SGM and the FFSM underestimate the cellular size and overestimate the acoustic concentration for the highest volume fractions, $\phi_{c} \geq 0.12$ (see red and green lines in Fig. 7). One can notice that the radius estimated by the classical models decreases as the cellular volume fraction increases, whereas the radius is generally estimated around $8.18 \mu \mathrm{m}$ when using the SFM, whatever the studied volume fraction. Only the acoustic concentrations estimated with the SFM agree well with the expected acoustic concentration whatever the volume fractions tested with relative errors between $6 \%$ and $19 \%$ (see magenta and blue lines in Fig. 7). Note that the SFM slightly underestimates the acoustic concentration and this can be explained by the overestimation of the scatterer radius (with relative errors between $22 \%$ and $47 \%$ ). We also computed the goodness-of-fit statistic of each model to the experimental data $B S C_{\text {meas }}$ (as defined in Eq. 

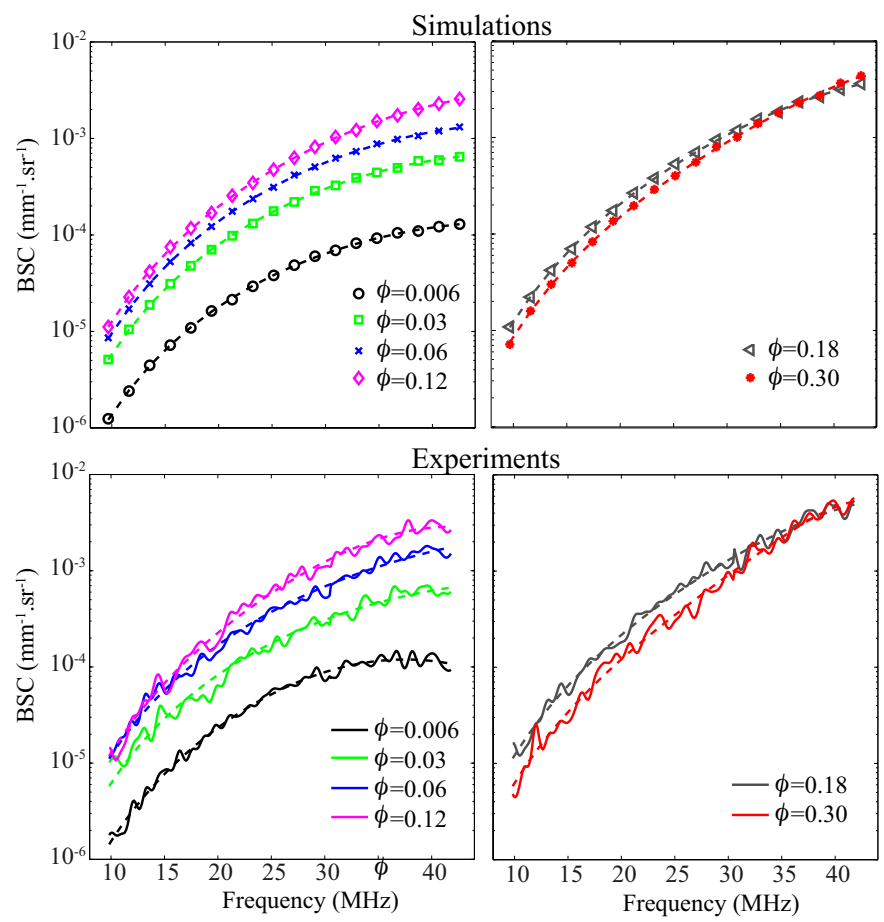

Fig. 4. Top row: Simulated BSCs obtained using Eq. (9) (symbols) and corresponding fitted curves (dashed lines) obtained with the polydisperse and the monodisperse SFM using estimator 1. Bottom row: Experimental measured BSCs from K562 cell pellet biophantoms (solid lines) and corresponding fitted curves (dashed lines) obtained with the polydisperse and the monodisperse SFM using estimator 1. Note that the fitted curves obtained with the polydisperse and the monodisperse SFM are superimposed.

(2) in Ref. 12). The SFM provided the best fit to the data with goodness-of-fit statistics approximatively equal to 0.94 for all the studied cell volume fractions (in comparison the goodness-of-fit statistics was between 0.77 and 0.85 for the SGM and FFSM).

\section{B. Comparison of the experimental and theoretical structure factors}

Figure 8 represents the simulated structure factors and the experimental structure factors obtained from the K562 cell pellet biophantoms at various cell volume fractions. One can observe that as the volume fraction increases, the structure factor curves become smoother. The simulated structure factor for the smallest volume fraction of 0.006 is approximately equal to 1 at all frequencies (i.e., comprised between 0.93 and 1.02), as expected.

The simulated and experimental structure factors were compared to two theoretical structure factors (Fig. 8): the polydisperse structure factor computed analytically as given in Eq. (7), and the monodisperse structure factor computed analytically as established in [25]. The theoretical structure factors use the K562 structural parameters ( $a=6.44 \mu \mathrm{m}$ and $\zeta=45$ ) for different volume fractions $\phi_{c}$ of $0.03,0.06,0.12,0.18,0.24$ and 0.30 . Since the product $k a$ is less than $1.12(0.26 \leq k a \leq 1.10$ in the $10-42 \mathrm{MHz}$ frequency range), the major peaks showed in Fig. 1 are not visible. The simulated structure factors correspond very well to the theoretical polydisperse modeling, as was 

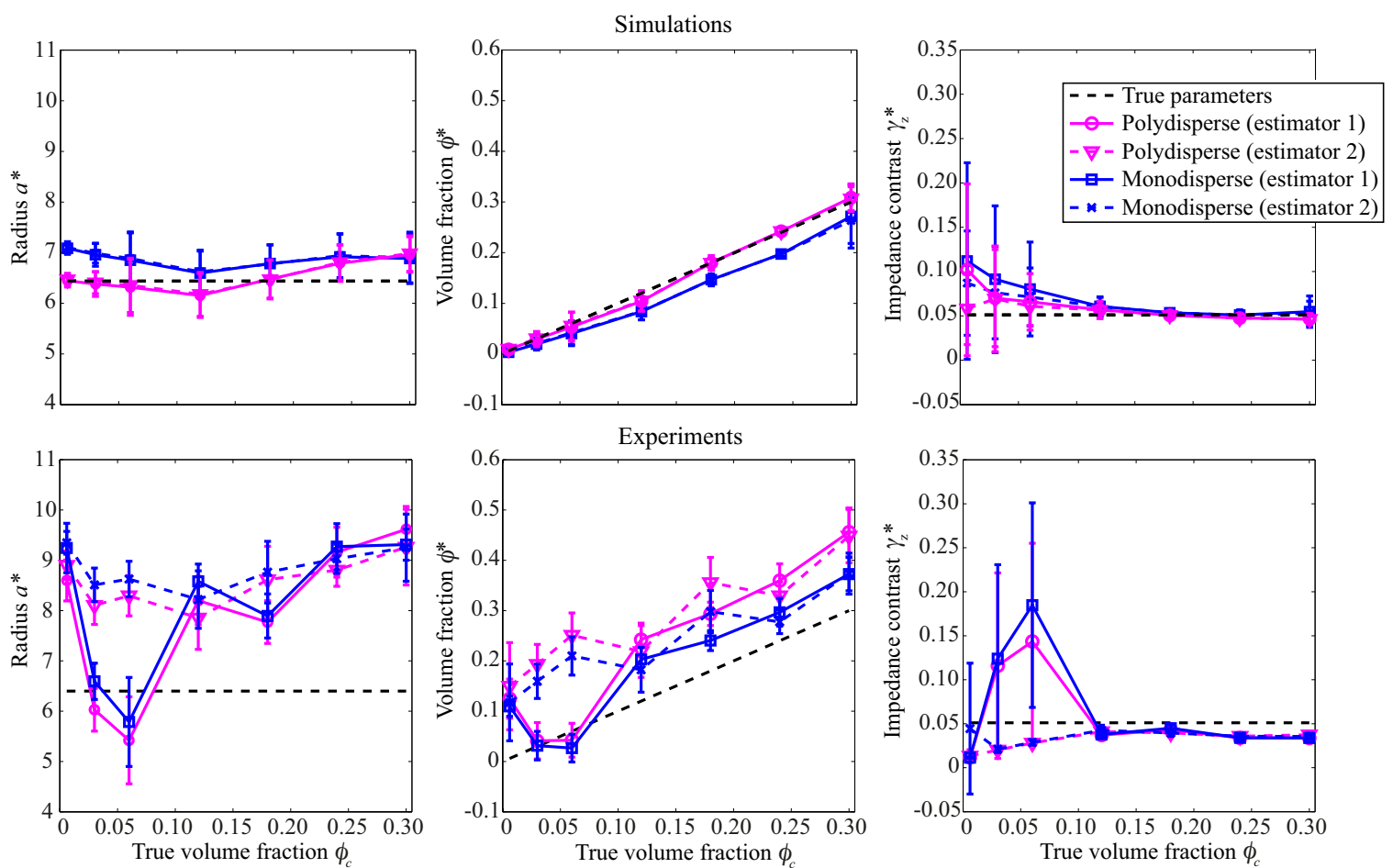

Fig. 5. Parameters $\left(a^{*}, \phi^{*}, \gamma_{z}^{*}\right)$ obtained from the inversion procedure based on the measured BSCs and estimated by the polydisperse or the monodisperse SFM using both estimators 1 and 2. Top row: results obtained from the simulated data. Bottom row: results obtained from the experimental data.

expected. The theoretical structure factors show good agreement $\left(R^{2} \geq 0.82\right)$ with the experimental structure factors for the cell volume fractions $\phi_{c} \geq 0.12$, but not for the two lowest volume fractions of 0.03 and 0.06 for which the correlation coefficients $R^{2}$ are less than 0.45 [see Fig. 8(b)]. Another noticeable difference is that the experimental curves present a larger magnitude for frequencies more than $35 \mathrm{MHz}$ in comparison with the theoretical curves in the majority of the studied volume fractions. The polydisperse modeling gives better results since the magnitudes of the theoretical structure functions are larger in the polydisperse case than in the monodisperse case, especially for concentrated media.

\section{QUS estimates from the experimental structure factors}

Figure 9 shows the estimated structural parameters $a^{*}, \phi^{*}$ and $\gamma_{z}^{*}$ using the method described in section III-D. Both monodisperse and polydisperse modeling give similar results. QUS parameters estimated from the simulated data agree very well with the true QUS parameters, except for the smallest true volume fraction of 0.006 for which the impedance contrast was overestimated. The structural parameters $\left(a^{*}, \phi^{*}\right)$ obtained from the experimental data give relative absolute errors of less than $30 \%$, except for the true cell volume fractions of 0.12 and 0.18 for which the relative absolute error is around $43 \%$ for the volume fraction estimation. For the experimental data, the impedance 


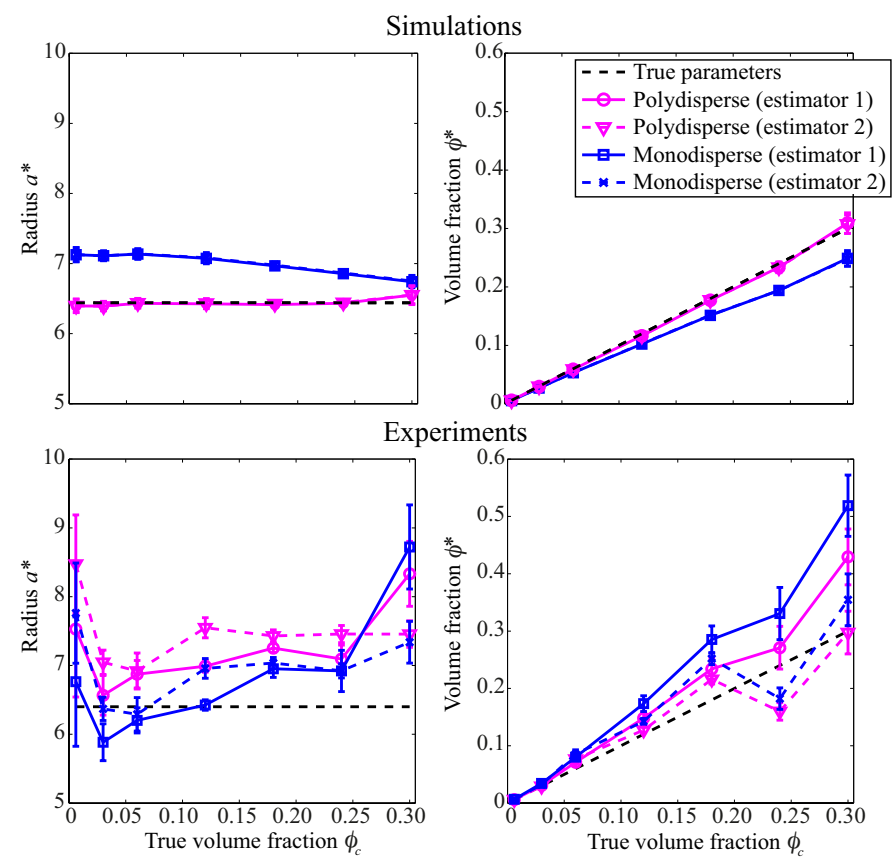

Fig. 6. Parameters $\left(a^{*}, \phi^{*}\right)$ obtained from the inversion procedure based on the measured BSCs using the polydisperse or monodisperse SFM when the relative impedance contrast is assumed to be known a priori $\left(\gamma_{z}=0.051\right)$. Top row: results obtained from the simulated data. Bottom row: results obtained from the experimental data.

contrast $\gamma_{z}^{*}$ is estimated between 0.019 and 0.060 , which is in a reasonable range of values when compared to the expected impedance contrast of 0.051 estimated by ultrasound using a specific inversion procedure in our previous study [23, Table II line 3].

\section{DISCUSSION AND CONCLUSION}

\section{A. Comparison of the SGM, FFSM and SFM}

Three scattering models, namely the SGM, the FFSM and the SFM, were examined to estimate scatterer size and acoustic concentration, as shown in Fig. 7. The SGM and the FFSM did not yield accurate structural parameters with relative errors more than $200 \%$ for the highest volume fractions $\phi_{c} \geq 0.12$, whereas the SFM permitted satisfactory estimation of the acoustic concentrations with relative errors between 6 and $19 \%$ for cell volume fractions ranging from 0.006 to 0.30 , i.e., for diluted and concentrated cell pellet biophantoms. In addition, the ability of the SFM to estimate three parameters (i.e., the scatterer size, volume fraction and relative impedance contrast) was assessed by using two approaches: a direct approach which consisted of fitting the measured $B S C_{\text {meas }}$ to estimate simultaneously the three parameters, and a combined approach which consisted of estimating the parameters $a^{*}$ and $\phi^{*}$ from the experimental structure factor, and then estimating the corresponding relative impedance contrast $\gamma_{\mathrm{z}}^{*}$ from the measured $B S C_{\text {meas }}$. The direct approach based on the simulated $B S C_{\text {sim }}$ gave similar estimates whatever estimator was used (top row of Fig. 5), whereas the results based on the measured $B S C_{\text {meas }}$ were dependent on the tested 

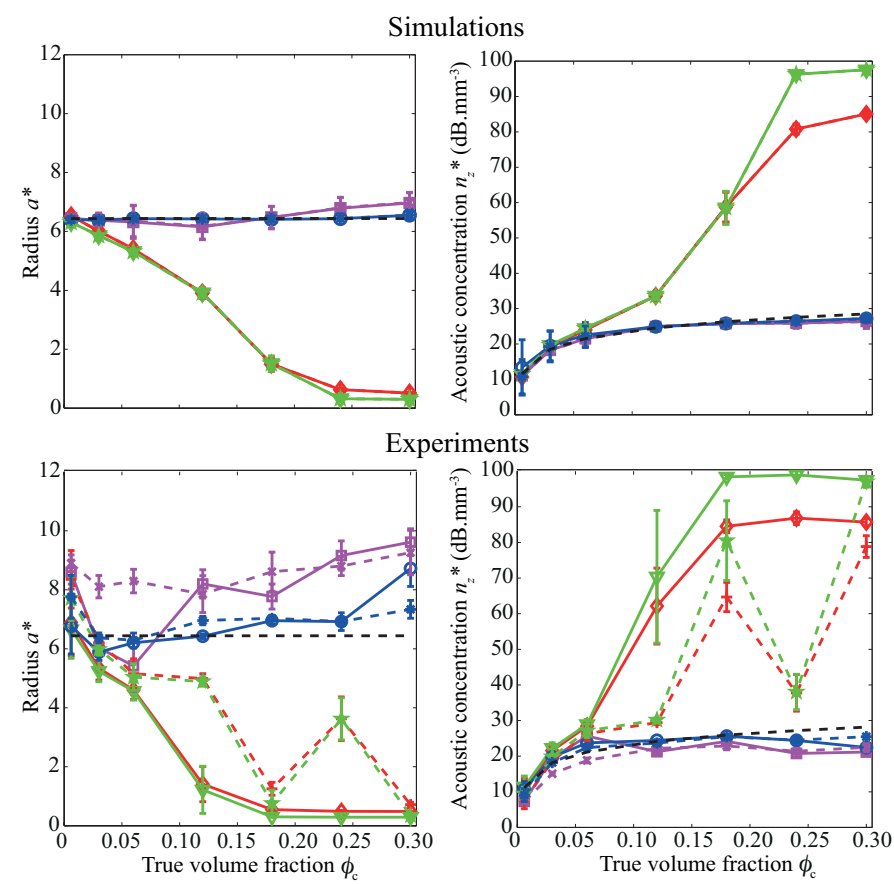

$$
\begin{aligned}
& \text { - - - True parameters } \square \text { SFM (Est. 1) } \\
& \neg-\text { SGM (Est. 1) - }-x-\text { SFM (Est. 2) } \\
& \begin{array}{ll}
-+- \text { SGM (Est. 2) } & -x-\text { SFM (Est. 2) } \\
-\nabla \text { FFSM (Est. 1) } & -0-\text { SFM }(\gamma=0.051 \text {,Est. 1) }
\end{array} \\
& -\star-\text { FFSM (Est. 2) - SFM }\left(\gamma_{z}^{\prime}=0.051\right. \text {,Est. 2) }
\end{aligned}
$$

Fig. 7. Radius $a^{*}$ and acoustic concentration $\left(n \gamma_{z}\right)^{*}$ estimated by the three scattering models (SGM, FFSM and SFM) using both estimators in the polydisperse case. Parameters obtained with the SFM when the impedance contrast is assumed to be known $a$ priori $\left(\gamma_{z}=0.051\right)$ are also plotted. Top row: results obtained from the simulated data. Bottom row: results obtained from the experimental data.

estimators, especially for the true smallest volume fractions $\left(\phi_{c} \leq 0.06\right)$ (bottom row of Fig. 5). When considering the experimental data, the direct approach yielded the best volume fraction estimates when using the monodisperse SFM (using estimators 1 or 2) for the true largest volume fraction (i.e., 0.24 and 0.30) with relative errors between $15 \%$ and $24 \%$. The use of the combined approach with the polydisperse or the monodisperse SFM resulted in better volume fraction estimates when compared to the direct approach, especially for the two smallest volume fractions (i.e., 0.03 and 0.06) with relative errors smaller than 30\% (as shown in Fig. 9). (Results obtained with the combined approach were even better for the simulated media with relative errors less than $11 \%$ for $a^{*}$ and $\phi^{*}$ at volume fractions ranging from 0.006 to 0.30 .)

\section{B. On the use of the comparison between sparse models and concentrated model}

Note that even if the SFM did not provide quantitatively satisfactory estimates for diluted media when the three QUS parameters are estimated simultaneously, the acoustic concentration estimated with the SFM matches well with the expected acoustic concentration for all the cell volume fractions studied using both estimators. The comparison 

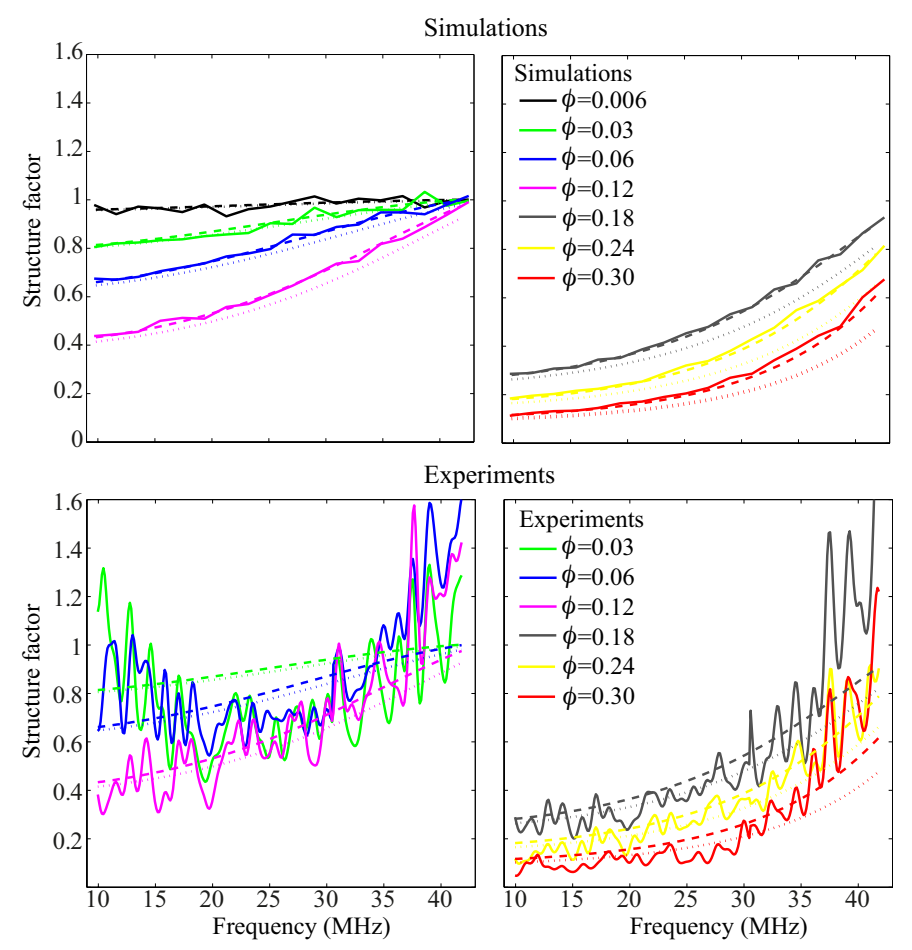

Fig. 8. Top row: simulated structure factors (in solid lines) as computed in Eq. (11). Bottom row: experimental structure factors (in solid lines) as computed in Eq. (15). Also represented are the theoretical structure factors (dotted lines for the monodisperse model and dashed lines for the polydisperse model) for the different volume fractions studied.

of FFSM, SGM and SFM demonstrates the superiority of the SFM for estimating the scatterer size and the acoustic concentration for diluted and concentrated media, as shown in Fig. 7. Since the three scattering models give similar QUS parameters for diluted media $\left(\phi_{c} \leq 0.12\right)$, one could compare the results from these three models in order to distinguish between diluted and concentrated media; if the structural parameters are similar with the SFM and a sparse scattering model (i.e., the SGM or the FFSM), then the medium can be considered to be diluted, and if the structural parameters differ, the medium can be considered to be concentrated. When a concentrated medium is identified, the simultaneous estimation of the three parameters with the SFM is reliable.

\section{Experimental and theoretical structure factor}

The discrepancies between the experimental and theoretical structure factors shown in Fig. 8(b) for frequencies higher than $35 \mathrm{MHz}$ (i.e., for a product $k a$ larger than 0.9) could be due to an inaccurate assumption on the cell shape in the polydisperse modeling. Indeed, the polydisperse structure factor is dependent on the scattering amplitude, derived here from the fluid-filled sphere form factor. The fluid-filled sphere assumption might be less reliable for modeling the shape and structure of cells, especially for a $k a$ value close to 1 . Moreover, the polydisperse modeling used herein remains isotropic and does not consider the polydispersity in terms of cell shape. In addition, inaccuracies in the expected parameters could contribute to the discrepancies. The mean radius and the gamma 

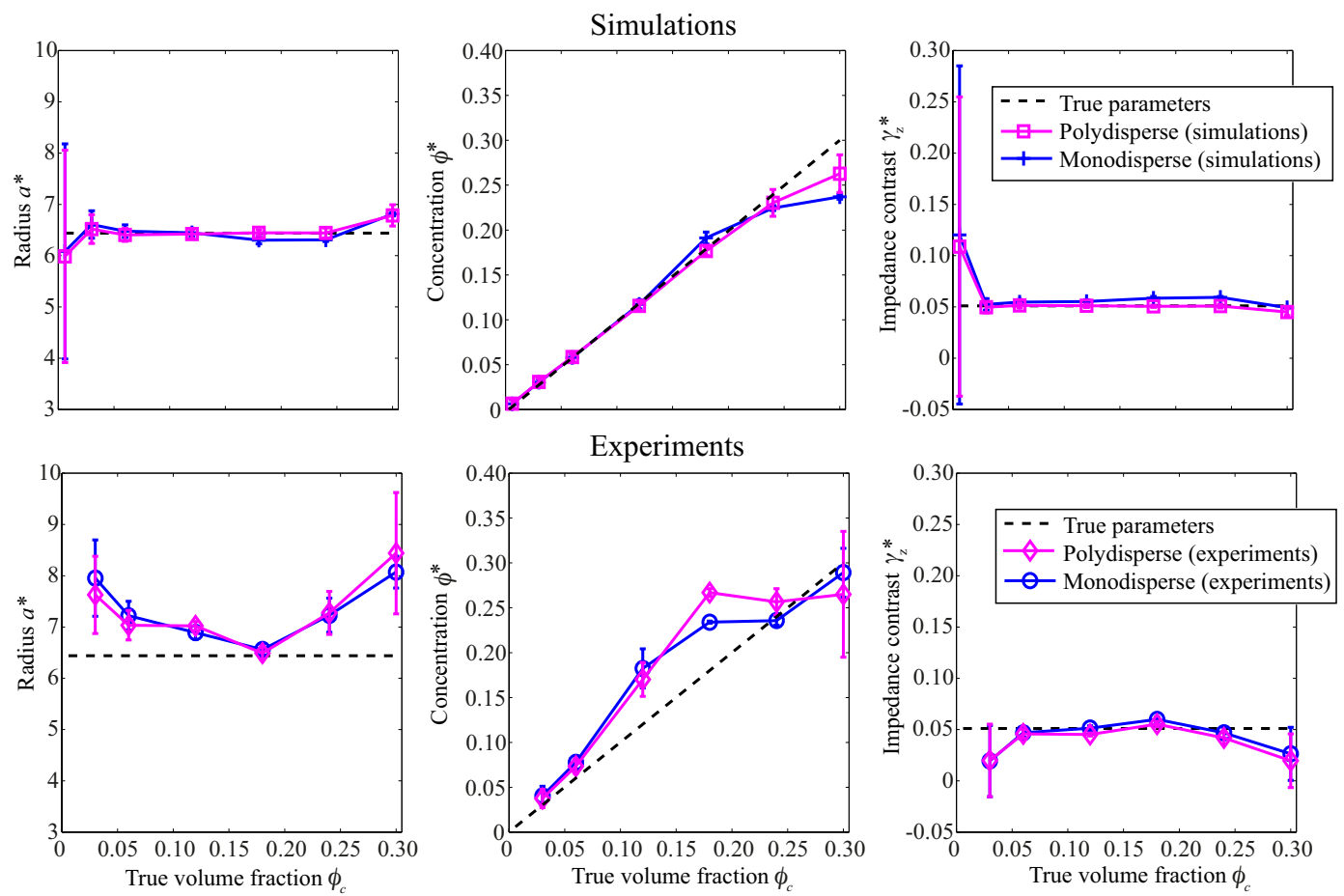

Fig. 9. Parameters $\left(a^{*}, \phi^{*}, \gamma_{z}^{*}\right)$ obtained from the inversion procedure based on the combined approach based on both structure factors and measured BSCs. Top row: results obtained from the simulated data. Bottom row: results obtained from the experimental data.

width factor were obtained by fitting the whole cell-size distribution estimated by optical microscope observation [23, Fig. 2] and if, for instance, the mean radius or the gamma width factor was underestimated, then the magnitude of the theoretical structure functions would be underestimated. Also, the true volume fraction values for cell pellet biophantoms used throughout the studies were estimated by using the Scepter 2.0 cell counter (Millipore, Molsheim, France) [23, section III.A] and could also have uncertainty.

Concerning the two lowest volume fractions of 0.03 and 0.06 with 0.006 being used as the reference in Eq. (15), the experimental structure factor curves look relatively distorted compared to the other curves, because of the lower SNR for the smallest volume fractions. The lower number of scatterers reduced the SNR by about 9 dB for the smallest volume fraction when compared to the larger volume fractions. Also, simulated and experimental structure functions become smoother as the volume fraction increases (Fig. 8). This can be attributed to the increase in scatterer number density as the volume fraction increases (because the ensemble averaging is performed on a much larger number of phasors associated with the scatterer locations). In this study and for the volume fractions evaluated, the simulated structure functions were computed by averaging over 1000 realizations, and the experimental structure functions were obtained from approximately 100 independent backscattered signals. To obtain smoother curves at low volume fractions, the number of realizations for the simulated data (or the number of backscattered signals from different regions-of-interest for the experimental data) must be increased. 


\section{Estimator choice}

Two estimators were studied to obtain the QUS parameters when using the direct method based on the fitting of the measured $B S C_{\text {meas }}$ [see Eqs. (13) and (14)]. As shown in Fig. 2, the cost function behaviors obtained by both estimators for the same raw data are different. Investigators interested in using the SFM for clinical studies have to decide which estimator to use. On one side, estimator 2 puts a higher weight on absolute errors in the higher frequencies, but clearly from a numerical implementation standpoint, the cost function seems to be much better behaved with a clear attraction basin near the minimum. Nevertheless, a few criteria can be used to choose the estimator depending on the clinical situation. For example, in the studies presented here, the SNR was satisfactory and the frequency bandwidth used to fit the BSC was large (i.e., 10-42 MHz), therefore estimator 1 could be used to produce estimates enforcing good fidelity of the BSC at high frequencies. In in vivo clinical applications where independent data acquired from large tissue areas having stationary ultrasound scattering properties (for SNR improvement via spectral averaging) may be unavailable or where using two transducers (for larger fitting bandwidth) may be unpractical, estimator 2 should probably be used. Similarly, in an experimental situation where the noise is indeed white and independent of the BSC amplitude, the use of estimator 2 should be favored. In fact, this rationale probably explains why the basin of attraction shown in Figure 2c for estimator 2 is steeper than that of estimator 1. Another point to consider is what is hypothesized to be the scattering source, because one could weight frequencies in the cost functions according to the expected resonance peaks in the BSC. Finally, several novel methods exist to reduce the noise in the experimentally estimated BSC and as outlined above, the noise is an important factor in the choice of an estimator. For instance, angular compounding uses data acquired from the same tissue region but different angles of incidence to obtain more independent data (under the isotropic scattering assumption) [28], [29]. Finally, signal processing techniques such as multitaper or Welch's methods can be used to reduce noise in the estimated power spectra at the expense of longer computational time [30], [31].

\section{E. On the use of the structure factor model}

One of the characteristics of the SFM is the ability to estimate simultaneously three parameters: the radius $a$, the volume fraction $\phi$ and the relative impedance contrast $\gamma_{z}$. When using the monodisperse SFM, the frequency dependent BSC behavior depends on the product $\sigma_{b}(k, a) S(k, a, \phi)$, whereas it depends only on $\sigma_{b}(k, a)$ when using the classical models (SGM or FFSM). For concentrated media, the structure factor $S(k, a, \phi)$ strongly modifies the frequency dependence of the BSC; this could allow separately estimating the volume fraction and the impedance contrast. As expected, we found that the estimation of structural parameters is more robust for concentrated media because the two estimators provide similar volume fractions for $\phi_{c} \geq 0.12$, as shown in Fig. 5. However, when the medium is diluted, the structure factor is approximately equal to 1 at all frequencies, and the SFM cannot satisfactorily estimate the three parameters $a, \phi$ and $\gamma_{z}$. That is why the QUS parameter estimates are more dependent on the used estimator for the smallest volume fractions $\phi_{c} \leq 0.06$. Although both estimators provide a good fitting of curves to the measured $B S C_{\text {meas }}$, as shown in Fig. 2(e), the parameter estimations obtained by the two estimators are very different for the true volume fractions of 0.03 and 0.06 ; the estimated radii and volume 
fractions match quite well with the true parameters (with relative errors of less than $15 \%$ for the radius and less than $37 \%$ for the polydisperse SFM) together with the overestimation of the impedance contrast when using estimator 1, whereas the estimated radii and volume fractions are largely overestimated together with the underestimation of the impedance contrast when using estimator 2 . This phenomenon can be explained by the difficulty in simultaneously evaluating three parameters that can have the same effect on the BSC behavior as explained below. Using the SFM for $k a \leq 1$, an increase in $a$ has the effect of decreasing the spectral slope, whereas an increase in $\phi$ has the effect of increasing the spectral slope, as shown by the plot of the structure factor in Fig. 1. That is why an overestimation of $\phi$ leads to an overestimation of $a$ and the overestimation of $a$ leads to underestimation of $\gamma_{z}$.

One could also observe that the parameter estimation procedure was limited to three parameters $\left(a, \phi\right.$ and $\left.\gamma_{z}\right)$ when considering the SFM. For the particular case of the polydisperse SFM, the gamma width factor $\zeta$ was assumed to be known a priori in order to reduce the number of unknown parameters. Indeed, when the simultaneous estimation of the four parameters $\left(a, \zeta, \phi\right.$ and $\gamma_{z}$ ) was performed using the polydisperse SFM (data not shown), the global minimum could show either an overestimation of $\zeta$ for which the parameter estimates are close to the estimates obtained with the monodisperse SFM, or an underestimation of $\zeta$ which could bring large relative errors (greater than 200\%) on the other estimates (data not shown). The present study shows that the use of the monodisperse SFM or the polydisperse SFM (when $\zeta$ is known a priori) gave similar results for the studied structural parameters ( $a=6.44 \mu \mathrm{m}, \zeta=45$ and $0.006 \leq \phi \leq 0.30$ ) when the product $k a$ is less than 1.1 (Fig. 5). Therefore, one could arguably use the monodisperse SFM for the estimation of structural parameters in in vivo clinical applications. However, further studies are required to determine the estimate biases when using the monodisperse SFM for wider size distribution $(\zeta<10)$ and for greater volume fractions $(\phi>0.30)$.

Another topic of interest is the robustness of the SFM when used on real tumors. Cell pellet biophantoms are probably a reasonable approximation of real tissues within uniform regions; for instance, a recent study demonstrated that the BSC of a homogeneous tumor (i.e., with limited extracellular matrix and no necrosis) is similar to that of a cell pellet biophantom of the same cell type [13]. At the moment, the SFM is definitively an improvement over the sparse models (FFSM and SGM) for modeling high cellular content in simple tumor composed of a single cell line. However, tumors can have very complex structures (e.g., extracellular matrix, several cell lines, microvasculature, etc) that may play a role in tumor backscatter [13]. In particular, sources of coherent scattering (e.g., periodically nonrandomly located scatterers and specular reflectors coming from blood vessels or organ boundaries) invalidate the assumption of uniformly randomly distributed scatterers and can significantly affect the QUS parameters estimated with the SFM. To reduce the effect of coherent scattering, Luchies et al. [32] proposed a segmentation technique in order to identify and remove the non-diffuse echoes from the QUS analysis. The technique proposed in [32] can be used to detect data blocks with non-diffuse echoes and remove them for the QUS analysis using the SFM. 


\section{F. On the bias and variance of estimates obtained using SFM}

The goal of the QUS estimates obtained using the SFM approach is to ultimately characterize tissue within the framework of a clinical application (e.g., cancer detection or staging). Our studies strongly support the idea that the use of simple SGM can yield tremendous errors when coherent scattering caused by dense medium is present (see Fig. 7), and could therefore mitigate any possible clinical benefits of QUS for the characterization of tumors/tissues with densely packed cells. In fact, the results indicate that even in the case of sparse media, our SFM-based approach provides better results. In particular, the bias of the radius and acoustic concentration estimates are much greater when SGM is used instead of SFM. In addition, the simulation results shown in the top rows of Figs. 5 and 7 demonstrate that biases and variances are very satisfactory for $a^{*}$ and $\phi^{*}$ : relative errors were less than $11 \%$ and the variance for $\phi^{*}$ equals $5 \times 10^{-5}$ for $\phi_{c}=0.006$ and is less than $8 \times 10^{-4}$ for $\phi_{c} \leq 0.03$. As expected, biases and variances obtained using the experimental data were larger than those obtained in the simulations, which can be attributed to small inaccuracies in the expected QUS parameters (such as the true mean radius or gamma width factor as discussed previously in V-C). Further study should be carried out to investigate theoretically the variance of QUS estimates with the SFM. Nevertheless, the simulation results already provide some insight on what is to be expected (top row of Fig. 5). For example, when the volume faction is low, the effects of the SFM on the BSC are limited because the structure factor is approximately equal to 1 at all frequencies. Therefore, we expect the variance to be strongly dependent on the volume fraction (i.e., variance increases when the volume fraction decreases); see, for example, the large variances obtained for the impedance contrast estimates for the three smallest true volume fractions (top row of Fig. 5). Finally, we believe that our estimates still have clinical value for tissue characterization because they show quantitative differences between different media. In fact, the experiments presented herein are a challenging case for an eventual clinical application of QUS, because the only assumed change between the investigated media is the volume fraction. For instance, if our SFM methods were to be used in the framework of cancer detection and characterization, we believe that in addition to a change in volume fraction, a change in scatterers could also occur providing another source of BSC contrast. This additional BSC contrast would most likely be misinterpreted by the SGM (or other sparse scattering models), while the SFM would be able to accurately separate these two sources of contrast.

\section{G. Concluding remarks}

In summary, this study demonstrated the satisfactory performance of the SFM even in the case of sparse media. Results also demonstrated the SFM superiority over sparse scattering models such as the SGM. This superiority was demonstrated not only by how much better the estimates agreed with the true value, but also in the improved goodness of fit between the experimental $B S C_{\text {meas }}$ and the theoretical $B S C_{\text {theo }}$ based on the SFM. Therefore, the methods described here could be used to obtain QUS estimates of tissue microstructure with better accuracy as well as provide better ultrasound tissue characterization tools. In general, histology photomicrographs of tumors and tissues often show densely packed cells. Thus, we can reasonably assume that the SFM is a more-appropriate 
TABLE I

ESTIMATION OF THE SCATTERER SIZE BASED ON THE INVERSION PROCEDURE BASED ON THE EXPERIMENTAL STRUCTURE FACTOR USING THE MONODISPERSE OR THE POLYDISPERSE ANALYTICAL STRUCTURE FACTOR FOR THE SIMULATED DATA (LINES 1 AND 2) AND FOR THE EXPERIMENTAL DATA (LINES 3 AND 4). THE* SYMBOL SIGNIFIES THAT THE SIZES ESTIMATED BY THE MONODISPERSE AND POLYDISPERE MODELS WERE STATISTICALLY SIGNIFICANTLY DIFFERENT (P-VALUE $<0.05)$.

\begin{tabular}{|c|c|c|c|c|c|c|c|c|c|}
\hline & & & $\phi_{c}=0.006$ & $\phi_{c}=0.03$ & $\phi_{c}=0.06$ & $\phi_{c}=0.12$ & $\phi_{c}=0.18$ & $\phi_{c}=0.24$ & $\phi_{c}=0.30$ \\
\hline 1 & Polydisperse & $a^{*}(\mu \mathrm{m})$ & $7.27 \pm 4.92$ & $6.21 \pm 0.43^{*}$ & $6.43 \pm 0.21^{*}$ & $6.34 \pm 0.11^{*}$ & $6.43 \pm 0.11^{*}$ & $6.46 \pm 0.05^{*}$ & $6.53 \pm 0.07^{*}$ \\
2 & Monodisperse & $a^{*}(\mu \mathrm{m})$ & $7.76 \pm 5.30$ & $6.72 \pm 0.45^{*}$ & $6.94 \pm 0.22^{*}$ & $6.83 \pm 0.10^{*}$ & $6.91 \pm 0.11^{*}$ & $6.94 \pm 0.05^{*}$ & $7.05 \pm 0.06^{*}$ \\
\hline 3 & Polydisperse & $a^{*}(\mu \mathrm{m})$ & - & $3.47 \pm 0.47$ & $6.94 \pm 0.24^{*}$ & $7.09 \pm 0.30^{*}$ & $7.13 \pm 0.11^{*}$ & $6.47 \pm 0.17^{*}$ & $6.99 \pm 0.12^{*}$ \\
4 & Monodisperse & $a^{*}(\mu \mathrm{m})$ & - & $3.33 \pm 1.16$ & $7.47 \pm 0.24^{*}$ & $7.62 \pm 0.31^{*}$ & $7.66 \pm 0.12^{*}$ & $7.00 \pm 0.17^{*}$ & $7.54 \pm 0.11^{*}$ \\
\hline
\end{tabular}

model to use for modeling densely packed cellular content in tumors and a wide range of other tissues. For future applications to soft tissue, the combined approach based on the experimental structure factors cannot be implemented in a clinical setting because of the unavailability of a reference BSC measurement on a diluted medium composed of the same cell line, but the direct SFM approach based on the measured $B S C_{\text {meas }}$ is original and promising. The QUS estimates derived from the direct approach with the SFM could better characterize tissue types and prove invaluable for assessing disease, detecting tumors, or monitoring treatment in vivo using clinical ultrasound systems.

\section{APPENDIX}

This appendix presents the estimates of the scatterer radius obtained from the experimental structure factor given in Eq. (15), assuming that the volume fraction (as well as the gamma width factor in the polydisperse inversion case) are known a priori.

For each studied volume fraction, the scatterer radius was determined by fitting the experimental structure factor $S_{\text {meas }}$ with the analytical monodisperse structure factor (or, with the analytical polydisperse structure factor), i.e., by minimizing the cost function:

$$
C(a)=\sum_{j}\left\|S_{\text {meas }}\left(k_{j}\right)-S_{\text {theo }}\left(k_{j}\right)\right\|^{2},
$$

over the frequency range from 10 to $42 \mathrm{MHz}$. Here also, optimization was performed by using "fminsearch". Empirical investigations revealed that the cost function has a unique global minimum as observed by plotting the cost function surfaces (data not shown).

Table I summarizes the mean scatterer size estimates for the simulated data (lines 1-2) and for the experimental data (lines 3-4). When using the polydisperse modeling, the estimated radius of the scatterer size was estimated between 6.21 and $6.53 \mu \mathrm{m}$ for $\phi_{c} \geq 0.03$ for the simulated data and between 6.47 and $7.13 \mu \mathrm{m}$ for $\phi_{c} \geq 0.06$ for the experimental data. So the radius estimates agree well with the true radius of the K562 whole cells, with a maximum relative error of $11 \%$. When using the monodisperse modeling, the radius estimates are slightly larger with a maximum relative error of $19 \%$. Comparison of radius estimates obtained from both polydisperse and 
monodisperse SFM using a t-test showed a statistically significant difference in radius value (p-value $<0.05$ ), except for the smallest volume fractions (as shown by the ${ }^{*}$ symbols in Table I). Table I also demonstrates that the polydisperse SFM was overall more accurate (i.e., statistically significantly smaller errors) in comparison with the monodisperse SFM. Note that the radius was largely overestimated for the true volume fraction of 0.006 in the simulated data, and largely underestimated for the true volume fraction of 0.03 in the experimental data. These wrong estimations may be explained by the distorted $S_{\text {sim }}$ or $S_{\text {meas }}$ curve for the lowest volume fractions that affect the data fitting.

\section{REFERENCES}

[1] F. L. Lizzi, M. Greenebaum, E. J. Feleppa, and M. Elbaum, "Theoretical framework for spectrum analysis in ultrasonic tissue characterization", J. Acoust. Soc. Am., vol. 73, no. 4, pp. 1366-1373, 1983.

[2] F. L. Lizzi, M. Ostromogilsky, E. J. Feleppa, M. C. Rorke, and M. M. Yaremko, "Relationship of ultrasonic spectral parameters to features of tissue microstructure", IEEE Trans. Ultrason. Ferroelect. Freq. Contr., vol. 33, no. 3, pp. 319-329, 1986.

[3] M. L. Oelze, J. F. Zachary, and W. D. O'Brien, "Characterization of tissue microstructure using ultrasonic backscatter: Theory and technique for optimization using a Gaussian form factor", J. Acoust. Soc. Am., vol. 112, no. 3, pp. 1202-1211, 2002.

[4] E. J. Feleppa, F. L. Lizzi, D. J. Coleman, and M. M. Yaremko, "Diagnostic spectrum analysis in ophthalmology: a physical perspective", Ultrasound Med. Biol., vol. 12, no. 8, pp. 623-631, 1986.

[5] E. J. Feleppa, T. Liu, A. Kalisz, M. C. Shao, N. Fleshner, and V. Reuter, "Ultrasonic spectral-parameter imaging of the prostate", Int. J. Imag. Syst. Technol., vol. 8, no. 1, pp. 11-25 1997.

[6] M. L. Oelze, W. D. O’Brien, J. P. Blue, and J. F. Zachary, "Differentiation and characterization of rat mammary fibroadenomas and 4T1 mouse carcinomas using quantitative ultrasound imaging", IEEE Trans. Med. Imaging, vol. 23, no. 6, pp. 764-771, 2004.

[7] J. Mamou, A. Coron, M. Hata, J. Machi, E. Yanagihara, P. Laugier, and E. Feleppa, "Three-dimensional high-frequency characterization of cancerous lymph nodes", Ultrasound Med. Biol., vol. 36, no. 3, pp. 361-375, 2010.

[8] J. Mamou, A. Coron, M. L. Oelze, E. Saegusa-Beecroft, M. Hata, P. Lee, J. Machi, E. Yanagihara, P. Laugier, and E. Feleppa, “Threedimensional high-frequency backscatter and envelope quantification of cancerous human lymph nodes", Ultrasound Med. Biol., vol. 37, no. 3, pp. 345-357, 2011.

[9] G. Ghoshal, R. J. Lavarello, J. P. Kemmerer, R. J. Miller, and M. L. Oelze, "Ex vivo study of quantitative ultrasound parameters in fatty rabbit livers", Ultrasound Med. Biol., vol. 38, no. 12, pp. 2238-48, 2012.

[10] S. C. Lin, E. Heba, T. Wolfson, B. Ang, A. Gamst, A. Han, J. W. Erdman, W. D. O’Brien, M. P. Andre, C. B. Sirlin, and R. Loomba, "Noninvasive diagnosis of nonalcoholic fatty liver disease and quantification of liver fat using a new quantitative ultrasound technique", Clin. Gastro. Hepatol., vol. 13, no. 7, pp. 1337-1345, 2015.

[11] R. J. Lavarello, W. R. Ridgway, S. S. Sarwate, and M. L. Oelze, "Characterization of thyroid cancer in mouse models using high-frequency quantitative ultrasound techniques", Ultrasound Med. Biol., vol. 39, no. 12, pp. 2333-41, 2013.

[12] M. L. Oelze and W. D. O'Brien, "Application of three scattering models to characterization of solid tumors in mice", Ultrasonic Imaging, vol. 28, no. 2, pp. 83-96, 2006

[13] A. Han, R. Abuhabsah, J. P. Blue, S. Sarwate, and W. D. O'Brien, "The measurement of ultrasound backscattering from cell pellet biophantoms and tumors ex vivo", J. Acoust. Soc. Am., vol. 134, no. 1, pp. 686-693, 2013.

[14] A. Han, R. Abuhabsah, J. P. Blue, S. Sarwate, and W. D. O'Brien, "Ultrasonic backscatter coefficient quantitative estimates from highconcentration Chinese hamster ovary cell pellet biophantoms", J. Acoust. Soc. Am., vol. 130, no. 6, pp. 4139-4147, 2011.

[15] M. Teisseire, A. Han, R. Abuhabsah, J. P. Blue, Jr., S. Sarwate, and W. D. O’Brien, "Ultrasonic backscatter coefficient quantitative estimates from Chinese hamster ovary cell pellet biophantoms", J. Acoust. Soc. Am., vol. 128, no. 5, pp. 3175-3180, 2010.

[16] R. M. Vlad, R. K. Saha, N. M. Alajez, S. Ranieari, G. J. Czarnota, and M. C. Kolios, "An increase in cellular size variance contributes to the increase in ultrasound backscatter during cell death", Ultrasound in Medicine \& Biology, vol. 36, no. 9, pp. 1546-1558, 2010.

[17] D. Savery and G. Cloutier, "A point process approach to assess the frequency dependence of ultrasound backscattering by aggregating red blood cells", J. Acoust. Soc. Am., vol. 110, no. 6, pp. 3252-3262, 2001. 
[18] E. Franceschini, R. K. Saha, and G. Cloutier, "Comparison of three scattering models for ultrasound blood characterization", IEEE Trans. Ultras. Ferroelectr. Freq. Control., vol. 60, no. 11, pp. 2321-2334, 2013.

[19] V. Twersky, "Low-frequency scattering by correlated distributions of randomly oriented particles", J. Acoust. Soc. Am., vol. 81, no. 5, pp. 1609-1618, 1987.

[20] E. Franceschini and R. Guillermin, "Experimental assessment of four ultrasound scattering models for characterizing concentrated tissuemimicking phantoms", J. Acoust. Soc. Am., vol. 132, no. 6, pp. 3735-3747, 2012.

[21] J. J. Faran, "Sound scattering by solid cylinders and spheres", J. Acoust. Soc. Amer, vol. 23, no. 4, pp. 405-418, 1951.

[22] A. Han and W. D. O'Brien, "Structure function for high-concentration biophantoms of polydiperse scatterer sizes", IEEE Trans. ultras. Ferroelectr. Freq. Control., vol. 62, no. 2, pp. 303-318, 2015.

[23] E. Franceschini, R. Guillermin, F. Tourniaire, S. Roffino, E. Lamy, and J.-F. Landrier, "Structure Factor Model for understanding the measured backscatter coefficients from concentrated cell pellet biophantoms", J. Acoust. Soc. Amer., vol. 135, no. 6, pp. 3620-3631, 2014.

[24] M. F. Insana and D. G. Brown, "Acoustic scattering theory applied to soft biological tissues", in Ultrasonic Scattering in Biological Tissues, edited by K. K. Shung and G. A. Thieme (CRC, Boca Raton, FL, 1993), Chap.4, pp. 76-124.

[25] M. S. Wertheim, "Exact solution of the Percus-Yevick integral equation for hard spheres", Physical Review Letters, vol. 10, no. 8, pp. 321-323, 1963.

[26] W. L. Griffith, R. Triolo, and A. L. Compere, "Analytical scattering function of a polydisperse Percus-Yevick fluid with Schulz distributed diameters", Phys. Rev. A, vol. 35, no. 5, pp. 2200-2206, 1987.

[27] R. K. Saha and G. Cloutier, "Monte Carlo study on ultrasound backscattering by three-dimensional distributions of red blood cells", Phys. Rev. E, vol. 78, 061919 pp. 1-9, 2008.

[28] A. L. Gerig, T. Varghese and J. A. Zagzebski, "Improved parametric imaging of scatterer size estimates using angular compounding", IEEE Trans. Ultras. Ferroelectr. Freq. Control., vol. 51, no. 6, pp. 708-715, 2004.

[29] R. Lavarello, J. Sanchez and M. Oelze, "Improving the quality of QUS imaging using full angular spatial compounding", Proc. IEEE Ultrasonics Symp., pp. 32-35, 2008.

[30] G. Ghoshal and M. L. Oelze, "Improved scatterer property estimates from ultrasound backscatter using gate-edge correction and a pseudoWelch technique", IEEE Trans. Ultras. Ferroelectr. Freq. Control., vol. 57, no. 12, pp. 2828-2832, 2010.

[31] I. M. Rosado-Mendez, T. J. Hall and J. A. Zagzebski, "Performance of an adaptive multitaper method for reducing coherent noise in spectral analysis of ultrasound backscattered echoes", Proc. IEEE Ultrasonics Symp., pp. 429-432, 2013.

[32] A. C. Luchies, G. Ghoshal, W. D. O’Brien and M. L. Oelze, "Quantitative ultrasonic characterization of diffuse scatterers in the presence of structures that produce coherence echoes", IEEE Trans. Ultras. Ferroelectr. Freq. Control., vol. 59, no. 5, pp. 893-904, 2012.

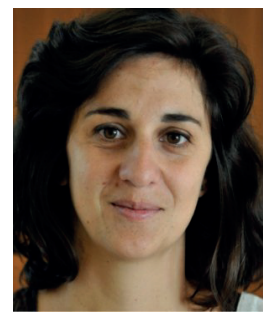

Emilie Franceschini received her M.S. degree in Acoustics from the University of Méditérannée, Marseille, 2003, and the Ph.D. degree in Acoustics from the University of Provence, Marseille, 2006. In 2007, she was a Postdoctoral Fellow at the Laboratory of Biorheology and Medical Ultrasonics, Research Center of the University of Montreal Hospital, Montreal, conducting research in ultrasonic blood characterization. Since October 2008, she is a Researcher at the French National Center for Scientific Research (CNRS) in the Laboratory of Mechanics and Acoustics CNRS UPR 7051, Marseille. Her research interests include ultrasound tissue characterization, ultrasound tomography, quantitative ultrasound techniques and inverse problems. She is a member of the French Acoustical Society (SFA), IEEE and IEEE

UFFC. 


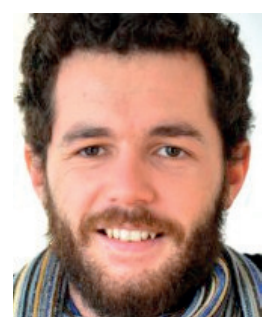

Romain de Monchy graduated from the Ecole Nationale Supérieure d'Arts et Métiers in 2013. He is currently pursuing a Ph.D. degree at the University of Aix-Marseille, Laboratory of Mechanics and Acoustics CNRS UPR 7051, Marseille. His research interests include quantitative ultrasound imaging and blood ultrasound characterization.

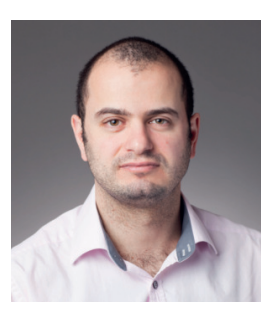

Jonathan Mamou was born in Saint-Germain-En-Laye, France in 1978. In July 2000, he graduated from the Ecole Nationale Supérieure des Télécommunications in Paris, France. In January 2001, he began his graduate studies in electrical and computer engineering at the University of Illinois at Urbana-Champaign, Urbana, IL. He received his M.S. and Ph.D. degrees in May 2002 and 2005, respectively. He is now Research Manager of the F. L. Lizzi Center for Biomedical Engineering at Riverside Research in New York, NY. His fields of interest include theoretical aspects of ultrasonic scattering, ultrasonic medical imaging, ultrasound contrast agents, and biomedical image processing. He is the co-editor of the book Quantitative Ultrasound in Soft Tissues published by Springer. Jonathan Mamou is a Senior Member of IEEE, a Fellow Member of the American Institute of Ultrasound in Medicine, and a Member of the Acoustical Society of America. Dr. Mamou currently serves as the Chair of the AIUM High-Frequency Clinical and Preclinical Imaging Community of Practice. He is an Associate Editor for Ultrasonic Imaging and the IEEE Transactions on Ultrasonics, Ferroelectrics, and Frequency Control and a reviewer for numerous journals. 